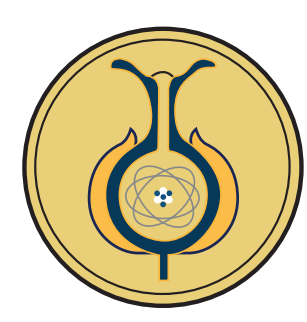

\title{
Análisis cromático y espectral de lacas acrílicas para retoque de pintura automotriz blanca mediante Reconocimiento de Pautas no Supervisado
}

\section{Spectral and chromatic analysis of automotive acrylic lacquers for white paint touch up using Unsupervised Pattern Recognition \\ Análise cromática e espectral de lacas acrílicas para retoque de pintura automotriz branca através do Reconhecimento de Pautas na Supervisão}

Judith Amador-Hernández judith.amador@uadec.edu.mx

Facultad de Ciencias Químicas,

Universidad Autónoma de Coahuila,

Saltillo Coahuila, México.

Orcid: http://orcid.org/0000-00031873-024X

\section{Miguel Velázquez-Manzanares} miguel_velazquez@uadec.edu.mx Facultad de Ciencias Químicas,

Universidad Autónoma de Coahuila,

Saltillo Coahuila, México.

Orcid: http://orcid.org/0000-00025125-1040
Edith Madaí Colunga-Urbina ecolunga@uadec.edu.mx

Facultad de Ciencias Químicas, Universidad Autónoma de Coahuila,

Saltillo Coahuila, México.

Orcid: http://orcid.org/0000-00027315-4903

\section{Iliana Margarita de la Garza-} Rodríguez

ilianagarza@uadec.edu.mx

Facultad de Ciencias Químicas, Universidad Autónoma de Coahuila,

Saltillo Coahuila, México.

Orcid: http://orcid.org/0000-00022064-5637

Recibido-Received: $2 / \mathbf{m a r} / 2018 \bullet$ Corregido-Corrected: 19/abr/2018.

Aceptado-Accepted: 18/jun/2018 • Publicado-Published: 31/ene/2019.

\section{Resumen}

En este trabajo, se caracterizó el color de lacas acrílicas blancas utilizadas para retocar pintura automotriz, mediante el espacio de color CIELab y el índice de blancura, utilizando el iluminante estándar D65 y el observador estándar a diez grados. A partir de tal información, fue posible reconocer las tendencias del color usado en este tipo de productos en el mercado nacional. Por otra parte, la aplicación de las técnicas quimiométricas de Análisis de Componentes Principales, Análisis de Grupos y Análisis Jerárquico, permitió reconocer semejanzas entre los colores observados, encontrando, entre otros, la mayor diferencia visual en la laca de blanco aperlado. Por otro lado, la laca de blanco Alaska de Mazda y la de blanco universal de Nissan fueron las más parecidas. También se concluye que el análisis cromático y el quimiométrico de datos espectrales fueron complementarios, por lo que se recomiendan ambos en el nivel de control de calidad. 
Palabras claves: color, CIELab; índice de blancura; pintura automotriz; quimiometría; lacas acrílicas.

Abstract

The color of white acrylic lacquers utilized to touch up automotive paint was characterized in this paper using the CIELab color space and the whiteness index, with standard illuminant D65 and the standard observer at ten degrees. Based on this information, the color trends used in this type of product in the national market were recognized. In addition, the chemometric techniques of the Principal Component Analysis, Cluster Analysis, and Hierarchical Analysis allowed for recognition of similarities between the observed colors, which found, among other aspects, the greatest visual difference in the pearl white lacquer. On the other hand, the Alaskan white lacquer from Mazda and the universal white lacquer from Nissan were the most similar. It is also concluded that the chromatic and chemometric analyses of spectral data complemented each other; therefore, both are recommended for quality control purposes.

Keywords: color; CIELab; whiteness index; automotive paint; chemometrics; acrylic lacquers.

\section{Resumo}

Neste trabalho, caracterizou-se a cor de lacas acrílicas brancas utilizadas para retocar pintura automotriz, através do espaço de cor CIELab e o índice de brancura, utilizando o iluminador padrão D65 e o observador padrão a 10 graus. A partir dessa informação, foi possível reconhecer as tendências da cor usada neste tipo de produtos no mercado nacional. Por outro lado, a aplicação das técnicas quimiométricas de Análise de Componentes Principais, Análise de Grupos e Análise Hierárquica permitiu reconhecer semelhanças entre as cores observadas, encontrando, entre outras, a maior diferença visual na laca de branco perolizado. Por sua vez, a laca do branco Alaska da MAZDA e a do branco universal da NISSAN foram as mais parecidas. Concluise, também, que a análise cromática e a quimiométrica de dados espectrais foram complementárias, razão pela qual, em nível de controle de qualidade, ambas são recomendadas.

Palavras-chaves: cor, CIELab; índice de brancura; pintura automotriz; quimiometria; lacas acrílicas.

$\mathrm{E}$ 1 color es producto de una apreciación subjetiva del entorno influenciada, entre otros, por las emociones, ya que el cerebro es el encargado de atribuir cualidades a objetos y ambientes, logrando así un rechazo o atracción hacia ellos (Kareklas et al., 2014; Schloss y Palmer, 2017; Best, 2017). Actualmente, se reconoce que cada color se asocia con un conjunto de emociones e ideas específicas, produciendo una sensación diferente en cada persona (Gong et al., 2017; Schloss, 2015). A lo largo del tiempo, se ha investigado la influencia que tienen los colores sobre las personas (Gómez y Herranz, 2011; Al-Ayash et al., 2016), llegando a las siguientes conclusiones generales:
- Colores cálidos. En ellos se agrupa al amarillo, rojo y todas sus combinaciones. Proporcionan alegría, calor e incluso son considerados excitantes.

- Colores fríos. Agrupan al azul, verde y sus combinaciones. Estos colores en tonos claros se caracterizan por brindar sensaciones de relajación o soledad, mientras que en matices oscuros orientan hacia la tristeza y melancolía (Ulusoy y Olguntürk, 2017).

Finalmente, debe destacarse que las preferencias de color residen en aspectos como la cultura, la edad, el sexo o incluso el tamaño del objeto por observar (Park, 2014; 
Prado-León, 2015; Cho et al., 2017a), por lo que su estudio es fundamental para atender las necesidades y preferencias de la sociedad actual (Lee et al., 2013; Michalski, 2014; Yu et al., 2017).

\section{Marco teórico}

Desde la perspectiva más simple, el color puede caracterizarse a través de tres valores, propiedades o atributos:

1. Tono. También conocido como color, tinte, matiz o valor. El tono es el color puro sin ningún tipo de alteración; es la manifestación pura de la radiación electromagnética de la región visible a una longitud de onda específica, o la resultante de la mezcla entre colores primarios, secundarios y terciarios.

2. Saturación. Es la cantidad de color aplicada sobre una superficie; varía de transparente a intenso en la medida en que se superponen capas de pigmento.

3. Luminosidad. Simulando la luz blanca, da la sensación de volumen y distancia; permite reproducir el fenómeno de brillo y oscuridad sobre un objeto (Mollica, 2013).

La medición de los atributos del color es factible gracias al desarrollo de sistemas basados en algoritmos matemáticos que describen cómo es que el ser humano logra percibir un color determinado. A estos modelos se les denomina espacios de color o sistemas de ordenamiento de color. Matemáticamente, están definidos por componentes vectoriales cuya combinación genera todos los posibles colores que pueden ser representados por ese espacio.
La Comisión Internacional de Iluminación (CIE, Commission Internationale de l'Eclairage) es la autoridad internacional responsable de establecer las normas que definen cada uno de los espacios de color, así como su interrelación. El espacio de color CIELab es una transformación no lineal del espacio XYZ, cuyas coordenadas son $L^{*}, a^{*}$ y b*, de acuerdo con la Figura 1 (Dubois, 2010). Este sistema se diseñó para que las diferencias en la percepción del color correspondieran a cambios equivalentes en estas coordenadas; actualmente se encuentran múltiples aplicaciones de dicho espacio en la literatura (Bouzidi et al., 2014; Feraru et al., 2015; Yi y Shamey, 2015).

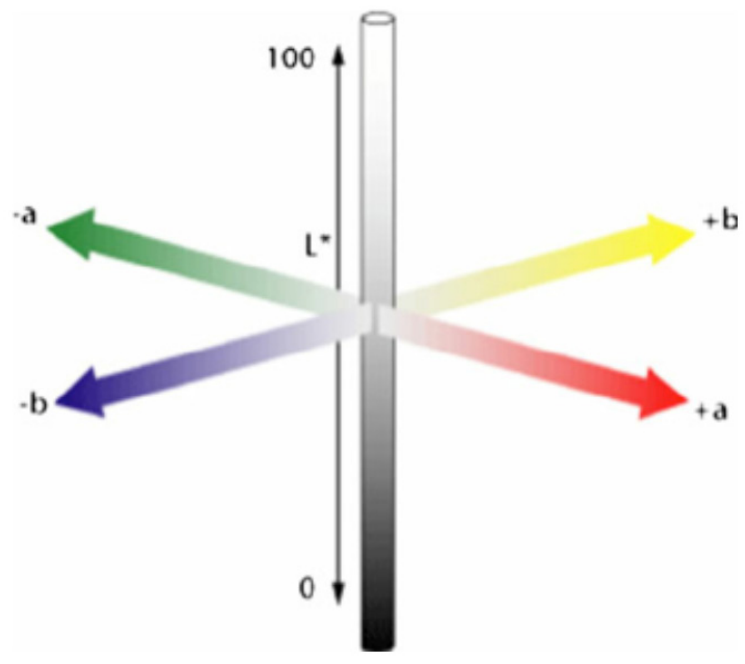

Figura 1. Espacio de color CIELab.

Tomada de https://mimoriarty.files.wordpress. com/2010/05/labmodel.jpg

Cabe destacar que, en este y otros espacios de color, el blanco se considera un caso particular que presenta el máximo grado de luminosidad, sin saturación ni tono (véase Figura 1, con $\mathrm{L}^{*}$ cercano a 100). El blanco encuentra un sinnúmero de aplicaciones comerciales, lo que ha llevado a la generación de múltiples tonalidades que requieren su 
caracterización, constituyendo así un caso de particular interés para los coloristas.

Un objeto blanco perfecto es aquel que refleja en su totalidad la radiación que incide sobre él en toda la región visible; es decir, no absorbe ni trasmite tal radiación. Si carece de brillo, recibe el nombre de difusor perfecto y presenta las siguientes coordenadas en el espacio de color Yxy:

$$
(\mathrm{Y}, \mathrm{x}, \mathrm{y})=\left(100, \mathrm{x}_{0}, \mathrm{y}_{0}\right)
$$

donde $\left(x_{0}, y_{0}\right)$ son las coordenadas del punto acromático para el iluminante correspondiente; es decir, $x$ y $y$ tienen valor cero y se encuentran sobre el eje central del espacio de color. Considérese que no hay un blanco perfecto único e invariable, ya que este depende directamente de las condiciones de iluminación (Puebla, 2006).

Sin embargo, existen objetos aparentemente blancos cuyas coordenadas $(x, y)$ no se encuentran en el eje del blanco-negro (condiciones acromáticas), sino que tienen una saturación particular para un tono azul. Para describir tales superficies, ha sido necesario introducir el término "blancura". De acuerdo con la norma E313-15 de la ASTM (2015), blancura es el atributo en la percepción del color de un objeto, según el cual este se aproxima al blanco preferido. Por su parte, el índice de blancura (WI, whiteness index) se ha definido como el número que indica el grado de concordancia del color de un objeto respecto al blanco preferido. Aunque existen varias expresiones matemáticas para su estimación, en la norma E313-15 de la ASTM se recomienda la propuesta por la CIE, siendo esta:

$$
\mathrm{WI}=\mathrm{Y}+(\mathrm{WI}, \mathrm{x})\left(\mathrm{x}_{\mathrm{n}}-\mathrm{x}\right)+(\mathrm{WI}, \mathrm{y})\left(\mathrm{y}_{\mathrm{n}}-\mathrm{y}\right)
$$

donde:
$Y, x, y=$ Factor de luminancia y coordenadas de cromaticidad del objeto bajo estudio.

$x_{\mathrm{n}} y y_{\mathrm{n}}=$ Coordenadas de cromaticidad del iluminante estándar de la CIE y la fuente usada.

$W I, x$ y $W I, y=$ Coeficientes numéricos tabulados.

El concepto de blancura es de interés tanto en el nivel de investigación (Vladusich, 2012; Cho et al., 2017b), como en el de su aplicación en caracterizar productos destinados al consumidor (Jung et al., 2012; Tutak et al., 2013; Kandi, 2013).

En este trabajo, se registraron los espectros de reflectancia difusa de ocho lacas acrílicas blancas utilizadas como retocador para pintura automotriz, a partir de los cuales se estimaron las coordenadas cromáticas en el espacio de color CIELab y el índice de blancura, utilizando el iluminante estándar D65 y el observador estándar a diez grados. La estimación de coordenadas cromáticas e índice de blancura permitió discutir las tendencias del color en este tipo de productos. Por otra parte, la aplicación de tres técnicas de Reconocimiento de Pautas no Supervisado para el análisis de los espectros de reflectancia difusa facilitó reconocer semejanzas entre las saturaciones observadas.

\section{Metodología}

\section{Instrumentación}

Se utilizó un espectrofotómetro UVVis (Cary 300, de Agilent Technologies), provisto de un accesorio de reflectancia difusa (DRA-CA-30i) y un estándar calibrado de reflectancia (Spectralon). Para el tratamiento de datos, se utilizó el software WinUV Color, de Agilent Technologies, 
Pirouette v. 3.11 de Infometrix v.4.5, así como Origin Pro de Originlab Corporation.

\section{Muestreo}

Las muestras analizadas se adquirieron en locales comerciales de la zona; su descripción se presenta en la Tabla 1. Las lacas acrílicas de tono blanco para retoque de pintura automotriz se depositaron como películas en portaobjetos, analizándose una vez que estuvieron secas.

\section{Procedimiento}

Los espectros de reflectancia difusa se adquirieron frente a un blanco de reflectancia en configuración de doble haz, en el intervalo de 360 a $830 \mathrm{~nm}$ con una resolución de $1 \mathrm{~nm}$, una integración de señal de $0.1 \mathrm{~s} \mathrm{y}$ rendija de $4 \mathrm{~nm}$.

Para la estimación de los atributos en los espacios de color, se utilizó el iluminante estándar D65, que semeja a la luz diurna media incluyendo la región ultravioleta con una temperatura de color correlativa de $6504 \mathrm{~K}$; el observador fue a 10 grados. A partir de estos elementos estándar, se registraron las coordenadas cromáticas en el espacio de color CIELab. También se estimó el índice de blancura de acuerdo con la norma E313-15 de la ASTM.

\section{Resultados y discusión}

En la Figura 2 se presentan los espectros de reflectancia difusa para las lacas acrílicas bajo estudio, mientras que en la Tabla 2 se integran los resultados del análisis cromático usando el espacio de color CIELab. Como puede observarse en la Figura 2, existen grandes similitudes espectrales con reflectancias alrededor del $70 \%$ en la región visible, condición requerida para considerarse un blanco; la excepción a esta tendencia la constituye la muestra con blanco aperlado, con reflectancias por debajo del $70 \%$ en todo el intervalo espectral de estudio.

Tabla 1

Muestras de lacas acrílicas de tono blanco, utilizadas como retocador para pintura automotriz

\begin{tabular}{cccc}
\hline Muestra & Nombre comercial & Modelo & Marca vehicular \\
\hline 1 & Blanco Alaska & Modelos 02-09 & Renault \\
2 & Blanco aperlado & Indefinidos & Universal \\
3 & Blanco brillante & Modelos 99-08 & Ford \\
4 & Blanco Candy & Indefinidos & Seat/Volkswagen \\
5 & Blanco marfil & Modelos 98-09 & Honda \\
6 & Blanco mármol & Modelos 01-09 & Mazda \\
7 & Blanco universal & Modelos 95-09 & Nissan \\
8 & Blanco polar & Modelos 98-09 & Volkswagen \\
\hline
\end{tabular}

Nota: Fuente propia de la investigación. 


\section{Análisis cromático}

En la Tabla 2 pueden observarse las coordenadas cromáticas en CIELab y el índice de blancura de las muestras bajo estudio. En general, las muestras se encuentran en el mismo cuadrante: luminosidad significativa $\left(\mathrm{L}^{*} \sim 70\right)$, con una tendencia apenas perceptible hacia el verde $\left(-\mathrm{a}^{*}\right)$ y amarillo $\left(+b^{*}\right)$; en ninguno de los casos se observan condiciones acromáticas $\left(\mathrm{a}^{*}=0, \mathrm{~b}^{*}=0\right)$. De acuerdo con estas tendencias, es evidente que se trata de blancos cálidos (con un ligero matiz de amarillo) y no blancos fríos (con ligero matiz de azul). Por otro lado, tales matices fueron apenas significativos, dado que todos se encontraron por debajo del 10 , en una escala de 0 a 100 .

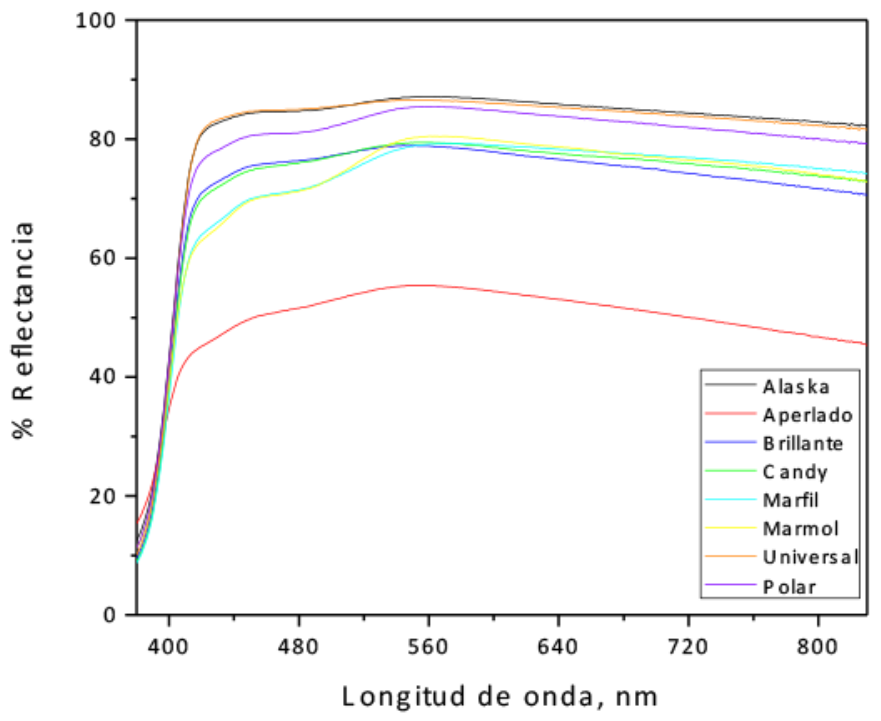

Figura 2. Espectros de reflectancia difusa de las lacas acrílicas bajo estudio. Nota: Fuente propia del estudio.

Cabe destacar que como muestra atípica se reconoció al blanco aperlado, con los valores más bajos de L* y de WI de la serie estudiada. Al observarse los espectros de reflectancia difusa, se determina que esta muestra es la que presenta también los valores más bajos de \% $\mathrm{R}$ en todo el intervalo espectral.

Los materiales presentan la apariencia de aperlados, u opalescentes, cuando radiación electromagnética de longitudes de onda cortas incide sobre su superficie y es dispersada de tal manera que se presenta una apariencia azulada respecto a la radiación reflejada y anaranjada/café respecto a la radiación transmitida, no absorbida; esto se debe a que su estructura presenta fases con distinto índice de refracción (Lee et al., 2005). Las pinturas metálicas para automóviles pueden presentar este tipo de apariencia cuando están constituidas por diversas capas a base de resina termoplástica, dióxido de titanio y un pigmento metálico o pseudometálico, con el que, además de lograrse el efecto metálico, se adquiere cierta opalescencia (Panush, 1993). En otras palabras, la presencia de partículas inmersas en una matriz, en la que los componentes tienen índice de refracción distinto, produce el efecto óptico de opalescencia. En el nivel cromático, disminuye la luminosidad del blanco porque una parte de la radiación también es transmitida y no solo reflejada, teniendo como consecuencia la reducción del WI; tal hecho se corrobora a través de la tendencia observada en el perfil del espectro de reflectancia difusa para esta muestra.

\section{Análisis quimiométrico}

Con la intención de establecer similitudes entre la cromaticidad de las lacas acrílicas, se aplicaron las siguientes técnicas quimiométricas pertenecientes al grupo de Reconocimiento de Pautas no Supervisado: Análisis de Componentes Principales (PCA), Análisis de Conglomerados por el método de las K-medias (CA), así como el Análisis Jerárquico. Inicialmente, se integró una matriz con las ocho muestras de laca, cada una representada por su espectro de 
Tabla 2

Coordenadas cromáticas en CIELab e índice de blancura de lacas para retoque de pintura automotriz, con iluminante d65 y observador a diez grados

\begin{tabular}{ccccc}
\hline \multirow{2}{*}{ Nombre de la muestra } & \multicolumn{3}{c}{ CIELab } & WI \\
\cline { 2 - 4 } & $\mathbf{a} \mathbf{A}^{*}$ & $\mathbf{b}^{*}$ & $\mathbf{L}$ & \\
\hline Blanco Alaska & $-0,79$ & 2,27 & 94,46 & 76,04 \\
Blanco aperlado & $-0,86$ & 6,87 & 90,39 & 44,61 \\
Blanco brillante & $-1,46$ & 3,70 & 90,94 & 60,91 \\
Blanco Candy & $-0,90$ & 1,79 & 94,314 & 77,85 \\
Blanco marfil & $-1,23$ & 7,85 & 90,78 & 40,89 \\
Blanco mármol & $-1,56$ & 2,83 & 90,76 & 64,60 \\
Blanco universal & $-2,12$ & 5,14 & 78,57 & 26,69 \\
Blanco polar & $-0,92$ & 3,59 & 93,52 & 67,65 \\
\hline
\end{tabular}

Nota: Fuente propia de la investigación.

reflectancia difusa consistente en señales de \% R a 470 longitudes de onda. Los datos fueron sometidos a un centrado sobre la media.

Para el PCA, se calcularon inicialmente los factores óptimos dirigidos a la construcción del modelo del sistema bajo estudio, partiendo de la tendencia observada en el PRESS de validación (Prediction Residual Error Sum of Squeares) en función del número de factores. El PRESS es un indicador de la capacidad de predicción del modelo, retirando iterativamente muestras de la serie para su estimación. De acuerdo con esta tendencia, el número de factores óptimo fue dos; la elección de más factores ya no redujo significativamente los errores, por lo que se corre el riesgo de sobreajuste, mientras que el uso de un solo factor condujo a un subajuste por el valor del PRESS registrado, más grande. El criterio de selección fue el mínimo local (López de Alba et al., 1997).
Con dos factores como óptimos, se construyó el modelo de PCA para identificar la distribución de scores en función de ellos. En otras palabras, se representaron los objetos de estudio en el plano de nuevas coordenadas dadas por los componentes principales o factores 1 y 2 . La aplicación posterior de la técnica de Análisis de Conglomerados, por el método de las K-medias a los scores estimados por PCA con dos factores, llevó a la asociación observada en la Gráfica 1, en la cual claramente puede distinguirse que la muestra de laca aperlada (u opalescente) es completamente distinta al resto de la serie, por ubicarse al lado izquierdo del eje del factor 1 , mientras que el resto de las muestras se encuentra en el lado opuesto de este. Recuérdese que el primer factor siempre incluirá información más significativa para describir el sistema bajo estudio que el segundo, y este más que el tercero, así sucesivamente. Un segundo grupo estuvo integrado por las muestras de laca 
mármol y marfil (apenas se distingue por la superposición de letras), cuyos espectros de reflectancia son prácticamente iguales (Figura 2). Un tercer grupo se integró por el resto de muestras.

La aseveración de que la muestra de laca aperlada es la más diferente de la serie puede corroborarse a través de la representación de la distancia de Mahalanobis en el análisis por PCA (Gráfica 2). Este parámetro matemático se utiliza en la identificación de objetos aberrantes u outliers, estimando distancias multivariables para identificar sus tendencias de distribución. Aunque con dos factores no se identifica la laca aperlada como un objeto aberrante, lo cierto es que se encuentra prácticamente en el límite (véase flecha en Gráfica 2).
Por otro lado, la visualización del poder de modelación en la Gráfica 3 permitió identificar que, para describir el sistema en el esquema de Componentes Principales, las variables más significativas fueron aquéllas comprendidas en el intervalo de 425 a 830 $\mathrm{nm}$, prácticamente toda la región visible del espectro electromagnético. Conforme el poder de modelación se acerca a 1, mayor es la importancia de la variable para describir el sistema; en este caso, tal indicador se encontró alrededor de 0,9 en el intervalo espectral antes señalado.

Posteriormente, se aplicó la técnica de Análisis Jerárquico para el estudio de los espectros de reflectancia difusa, utilizando la estrategia de asociación completa a partir de distancias euclidianas entre objetos. Los resultados de asociación se muestran en la Gráfica 4. Como puede apreciarse, la muestra de blanco aperlado no se asoció

\section{Gráfica 1}

Agrupación de muestras por PCA-CA, partiendo de los espectros de reflectancia difusa

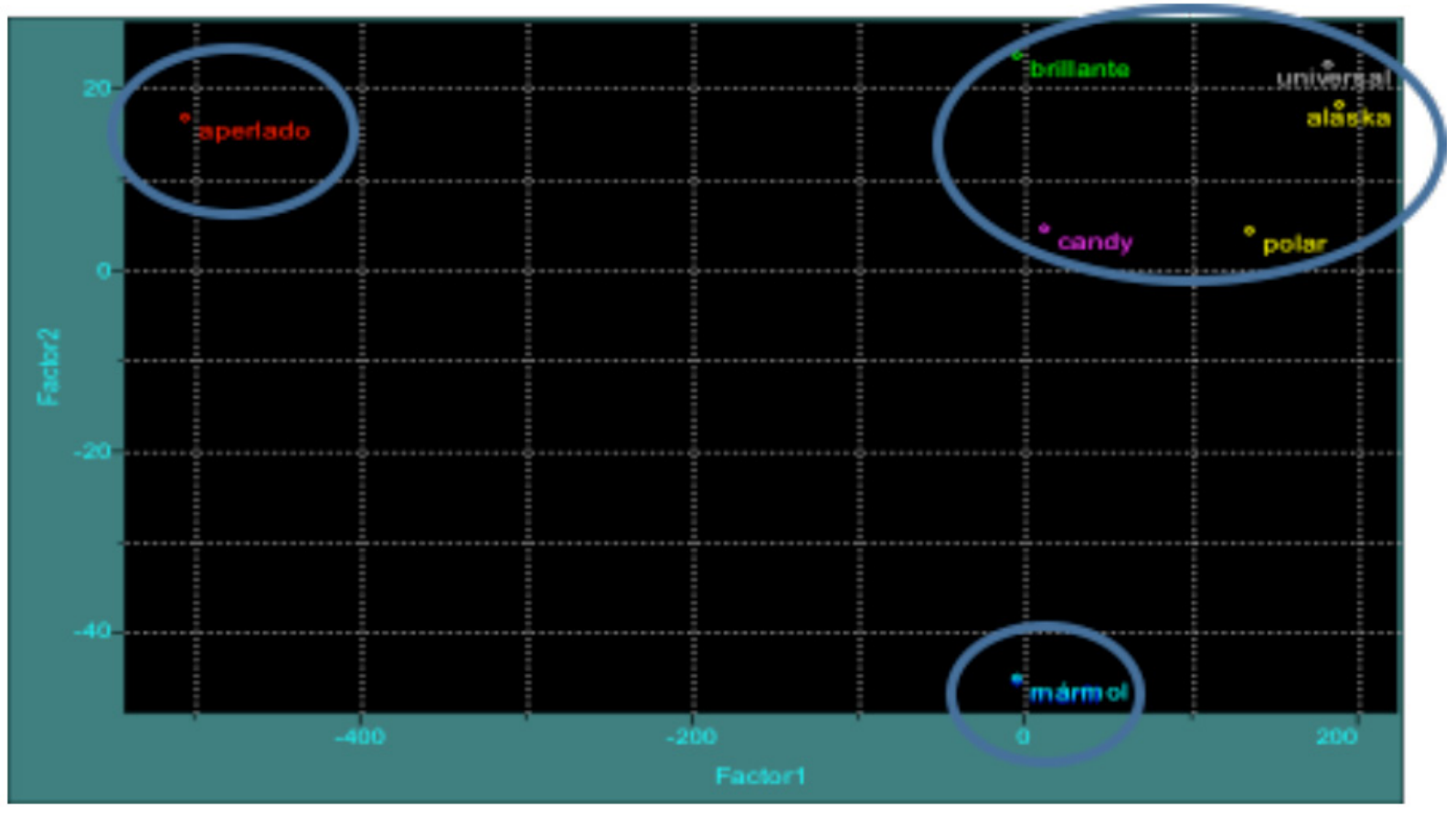

Nota: Fuente propia del estudio. 
con el resto (grado de similitud de cero). La muestra de blanco polar de Volkswagen fue la segunda en discrepar en la serie, aunque con un grado de similitud significativo a la par de los blancos universal y Alaska (alrededor del $90 \%$ ). Por otro lado, se encontró gran similitud entre los siguientes pares de lacas:

1. Blanco mármol de Mazda y blanco marfil de Honda (>90\%).

2. Blanco Alaska de Renault y blanco universal de Nissan (>90\%).

3. Blanco Candy de Seat y blanco brillante de Ford (>90\%).

Posteriormente, se aplicó la técnica PCA al análisis de los valores cromáticos del espacio de color CIELab (L*, a*, b*), así como al índice de blancura (WI). En este caso, se utilizó el autoescalado como pretratamiento de datos, debido a que cada variable tiene magnitudes diferentes. Al igual que en el estudio de espectros de reflectancia, se encontró que era necesario incluir dos factores para modelar la información.

En la Gráfica 5 se muestra la distribución de las lacas en función en el espacio definido por los factores 1 y 2 elegidos. La aplicación de la técnica de Análisis de Conglomerados por K-medias a los scores llevó a identificar también tres grupos, con los mismos integrantes en el análisis cromático y en el espectral, de acuerdo con la comparación de las gráficas 1 y 5 . Aunque las muestras de blanco mármol y blanco marfil se encontraron en el mismo grupo, no resultaron

\section{Gráfica 2}

Distancia de Mahalanobis para la identificación de muestras aberrantes en la serie de espectros de lacas analizadas por PCA

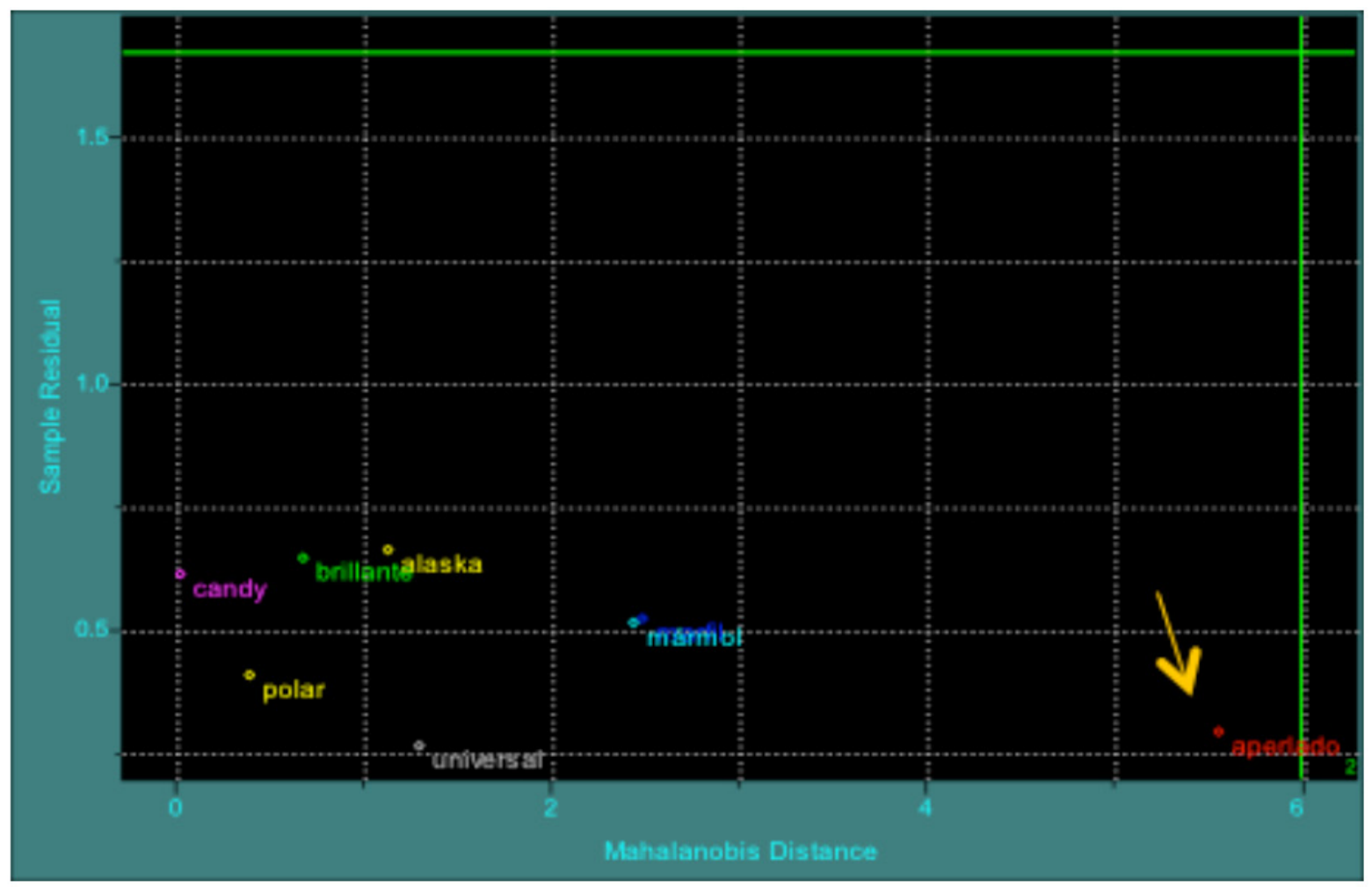

Nota: Fuente propia del estudio. 


\section{Gráfica 3}

Poder de modelación vs. variables espectrales utilizadas para describir las lacas acrílicas

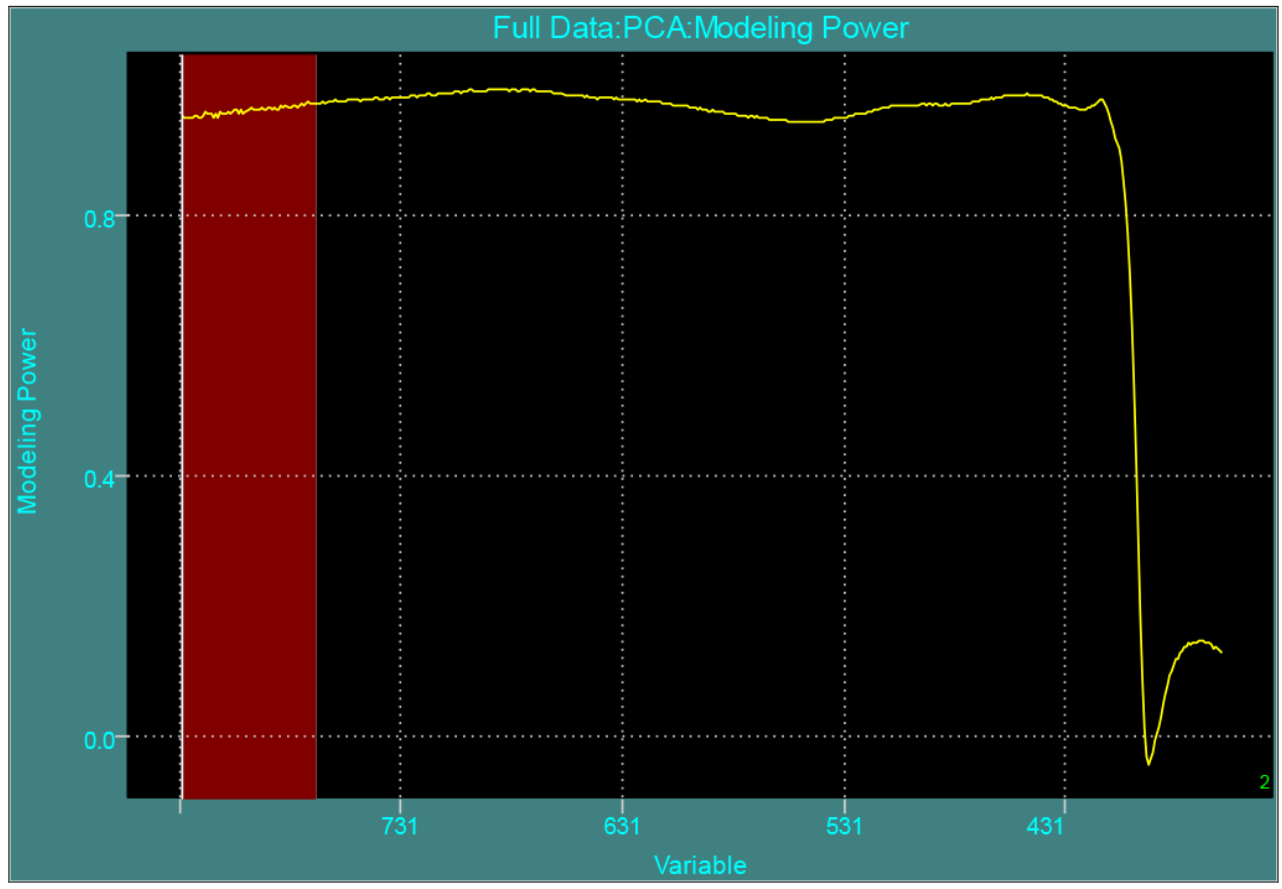

Nota: Fuente propia del estudio.

\section{Gráfica 4}

Análisis jerárquico de lacas para retoque de pinturas color blanco, a partir de datos espectrales

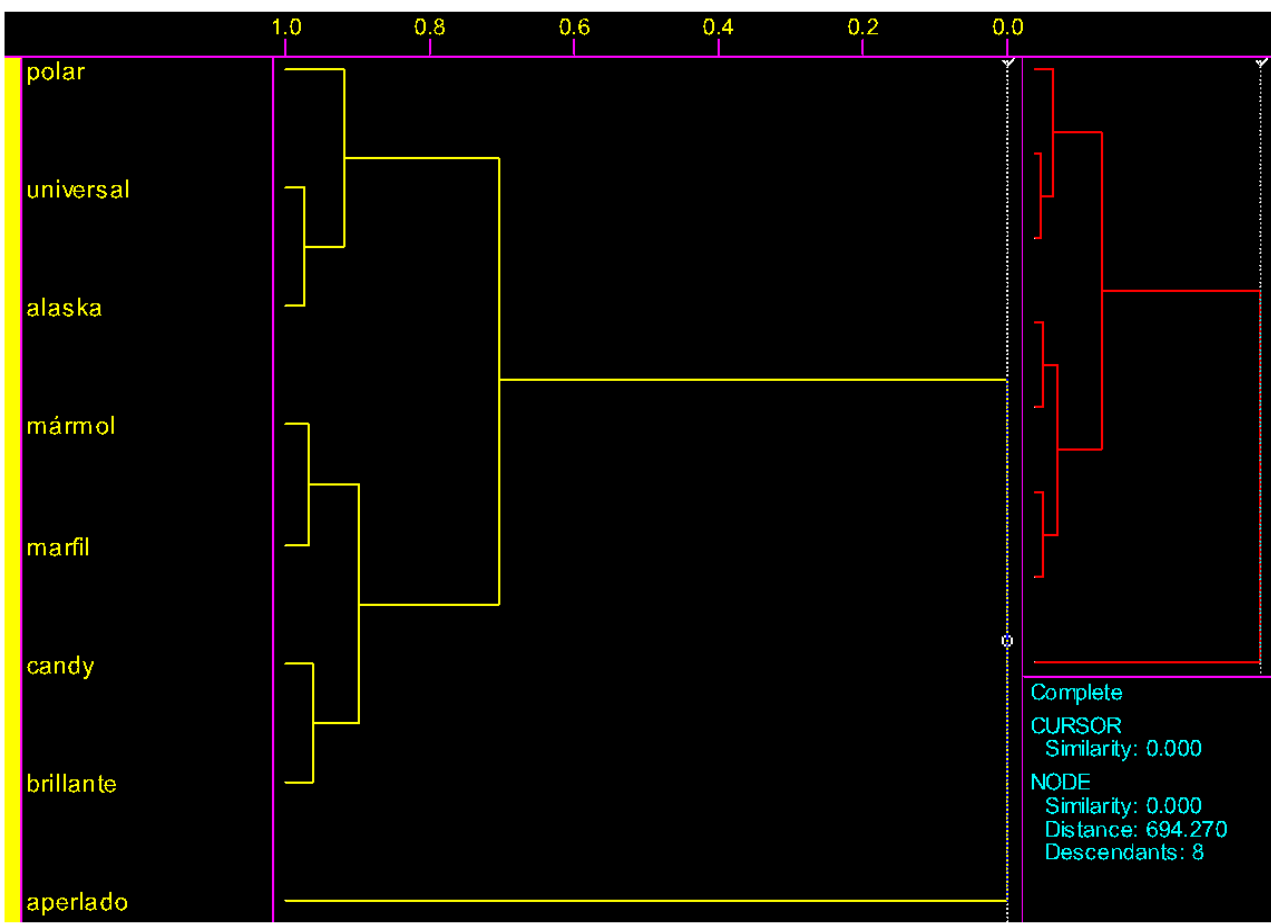

Nota: Fuente propia del estudio.

Artículo protegido por licencia Creative Commons: BY-NC-ND / Protected by Creative Commons: BY-NC-ND

Uniciencia es una revista de acceso abierto/ Uniciencia is an Open Access Journal. 


\section{Gráfica 5}

\section{Agrupación de lacas mediante PCA-CA, a partir de coordenadas cromáticas de CIELab y WI}

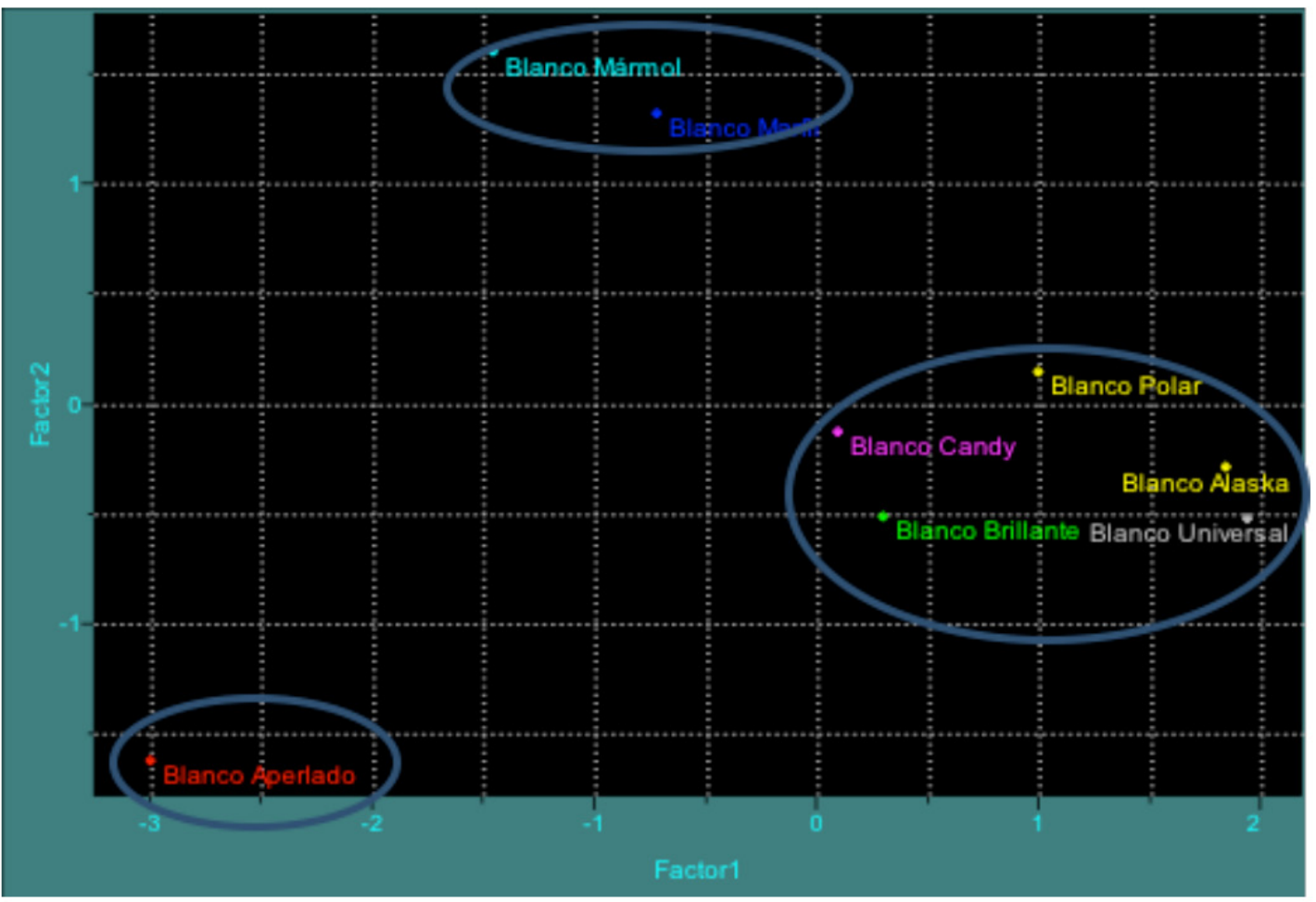

Nota: Fuente propia del estudio.

prácticamente iguales como en el caso del análisis de datos espectrales.

A partir de la distribución de los loadings o vectores de regresión en función del número de factores, pudo apreciarse que la variable $b^{*}$ fue determinante en la diferenciación de las muestras. Esta variable se asocia a la caracterización de los tonos amarillo y azul; valores de b+ indicaron una tendencia al amarillo, aunque en una escala mínima, pues sus valores fueron menores a diez en todos los casos, en una escala de 0 a 100. Se llegó a la misma conclusión sobre la importancia de $b^{*}$ para distinguir las lacas bajo estudio, a partir de la representación del poder de modelación en función de las variables de partida (Gráfica 6).

Finalmente, en la Gráfica 7 se presentan las similitudes entre muestras de laca encontradas por el Análisis Jerárquico, a partir de las coordenadas cromáticas en el espacio CIELab y WI. Igual que en la serie de datos anterior, se utilizó una asociación completa a partir de distancias euclidianas entre objetos.

De acuerdo con los resultados observados, las lacas más similares fueron las de color blanco Alaska de Mazda y blanco universal de Nissan, seguidas de las de blanco Candy de Seat y blanco brillante de Ford. La laca de blanco aperlado también se identificó como la más distinta de la serie, aunque se asoció al par de blanco mármol de Mazda y al blanco marfil de Honda, por una similitud mayor al $20 \%$. En general, la asociación de lacas por similitud fue casi la misma que la realizada a partir de los espectros de reflectancia, salvo en el caso de la muestra aperlada; sin embargo, el grado de similitud entre muestras varió, debido, probablemente, a 


\section{Gráfica 6}

Poder de modelación en PCA en función de las variables cromáticas utilizadas para describir las lacas acrílicas

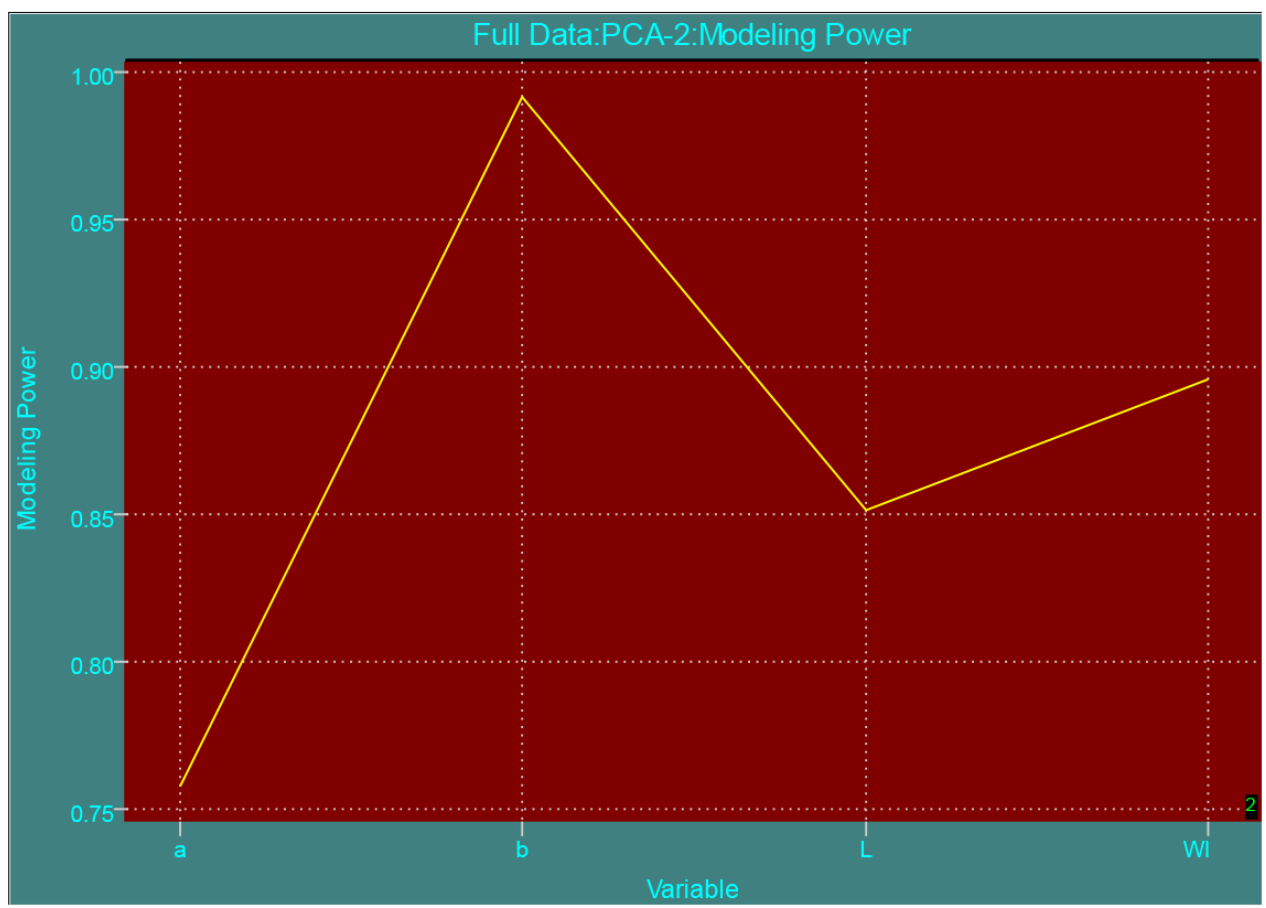

Nota: Fuente propia del estudio.

\section{Gráfica 7}

Análisis jerárquico de las lacas, utilizando datos cromáticos

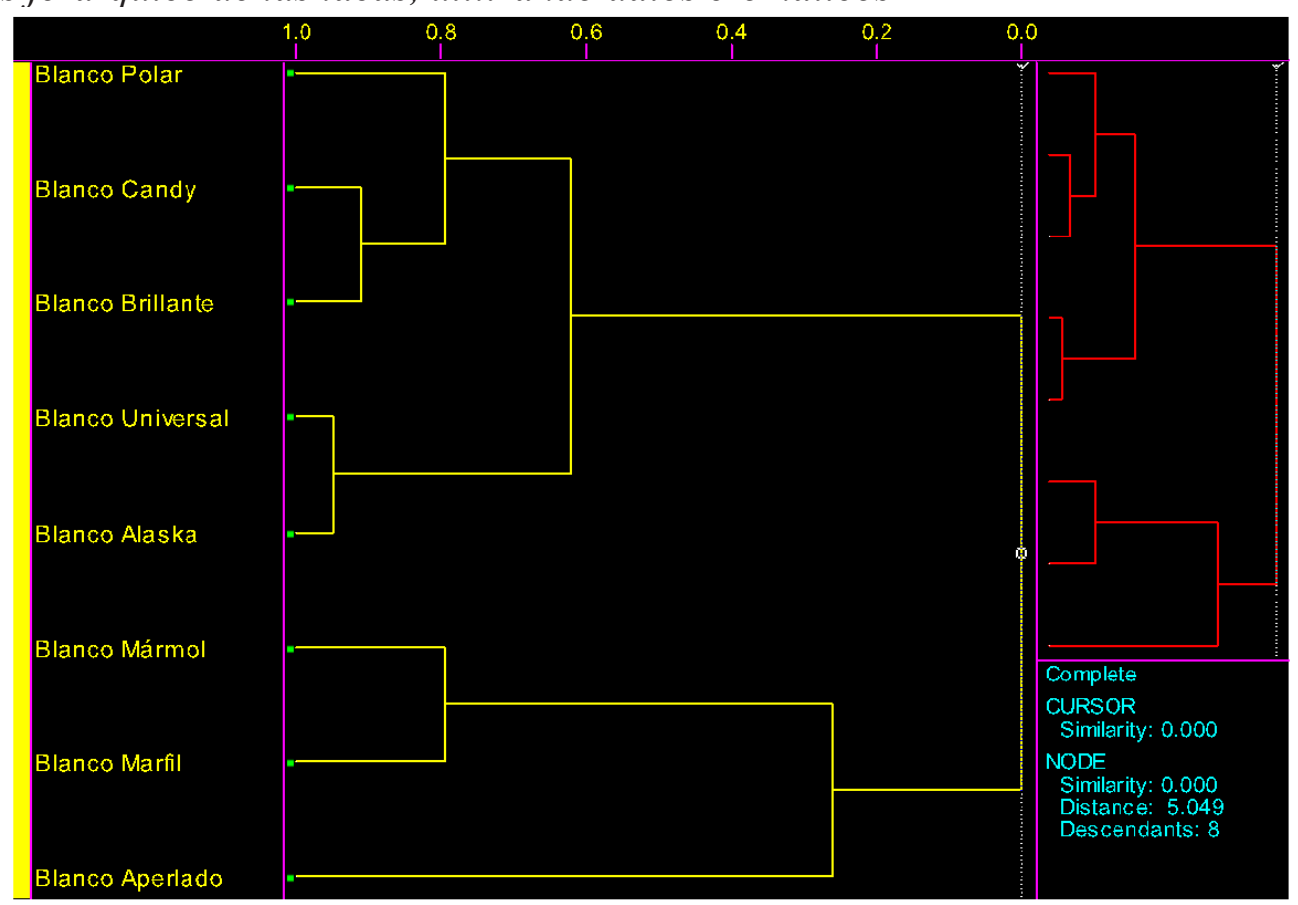

Nota: Fuente propia del estudio.

Judith Amador-Hernández, Miguel Velázquez-Manzanares, Edith Madaí Colunga-Urbina, Iliana Margarita de la Garza-Rodríguez y Diana Celene Sánchez-Hernández

Artículo protegido por licencia Creative Commons: BY-NC-ND / Protected by Creative Commons: BY-NC-ND

Uniciencia es una revista de acceso abierto/ Uniciencia is an Open Access Journal. 
que los efectos de iluminación no se consideran en el análisis espectral, pero sí en el análisis cromático.

\section{Conclusiones}

En este trabajo, se alcanzó exitosamente la caracterización de lacas para retoque de pintura automotriz, mediante análisis cromático y espectrometría de reflectancia difusa. Se reconoció una marcada tendencia hacia los blancos cálidos, con ligero matiz amarillo. Destaca la muestra de blanco aperlado, la cual manifestó valores cromáticos y espectrales muy distintos al resto de la serie, debido al fenómeno de opalescencia, donde su composición favoreció la transmitancia de parte de la radiación incidente en un medio con distintos índices de refracción.

Por otro lado, el análisis quimiométrico permitió reconocer semejanzas en los blancos observados, encontrándose, por ejemplo, que el blanco Alaska de Mazda y el blanco universal de Nissan fueron los más parecidos, por lo cual se pueden usar indistintamente. También se concluye que ambas técnicas fueron complementarias, lo que lleva a establecer la importancia de su monitoreo en el nivel de control de calidad y no solo del análisis cromático.

\section{Agradecimientos}

Se agradece a CONACYT el financiamiento otorgado para el presente trabajo (proyecto COAH-2013-C24-206199).

\section{Declaración}

Los autores manifiestan que no tienen ningún tipo de interés en favorecer o poner en desventaja alguna de las marcas mencionadas tras el análisis, así como que no se recibió apoyo económico que pueda ocasionar algún conflicto ético en los resultados del estudio.

\section{Referencias}

Al-Ayash, A.; Kane, R. T.; Smith, D. y Green-Armytage, P. (2016). The influence of color on student. Color Research and Application, 41(2), 96-205. DOI: 10.1002/col.21949.

ASTM E313-15e1. (2015). Standard practice for calculating yellowness and whiteness indices from instrumentally measured coordinates. USA: ASTM International. Recuperado de: https://www.astm.org/Standards/E313.htm

Best, J. (Ed.). (2017). Colour design: theories and applications, second edition. United Kingdom: Woodhead Publishing.

Bouzidi, N.; Siham, A.; Concha-Lozano, N.; Gaudon, P.; Janin, G.; Mahtout, L. y Merabet, D. (2014). Effect of chemico-mineralogical composition on color of natural and calcined kaolins. Color Research and Application, 39(5), 499-505. DOI: 10.1002/col.21813.

Cho, Y. J.; Ou, L. Ch. y Ronnier Luo. (2017a). A cross-cultural comparison of saturation, vividness, blackness and whiteness scales. Color Research and Application, 42(2), 203 2015. DOI: 10.1002/col.22065.

Cho, Y. J.; Ou, L. Ch.; Cui, G. y Luo, R. (2017b). New colour appearance scales for describing saturation, vividness, blackness, and whiteness. Color Research and Application, 42, 552-563. DOI: 10.1002/col.22114.

Dubois, E. (2010). The structure and properties of color spaces and the representation of color images. Synthesis Lectures on Image, Video, and Multimedia Processing, 4(1), 1-129. DOI: $\quad$ 10.2200/S00224ED1V01Y200910IVM011.

Feraru, D.; Mihaly, M. y Meghea, A. (2015). Chromatic analysis of blue ballpoint pen inks and related dyes. Color Research and Application, 40, 169-177. DOI: 10.1002/col.21866.

Gómez, B. y Herranz, A. (2011). La comunicación sin palabras. Barcelona, España: Marge Books.

Gong, R.; Wang, Q.; Hai, Y. y Shao, X. (2017). Investigation on factors to influence color emotion and color preference responses. Optik, 136, 71-78. DOI: 10.1016/j.ijleo.2017.02.026. 
Jung, H.; Suk, H. J.; Kitaguchi, S.; Sato, T. y Kajiwara, K. (2012). Color tolerance prediction for recycled paper based on consumers' awareness. Color Research and Application, 37(4), 272-280. DOI: 10.1002/col.20676.

Kandi, S. G. (2013). The effect of paper appearance on printed color of inkjet printer. Color Research and Application, 38(4), 284-291. DOI: 10.1002/col.21724.

Kareklas, I.; Brunel, F. F. y Coulter, R. A. (2014). Judgment is not color blind: The impact of automatic color preference on product and advertising preferences. Journal of Consumer Psychology, 24(1), 87-95. DOI: 10.1016/j. jcps.2013.09.005.

Lee, S. M.; Lee, K. T.; Lee, S. W. y Song, J. K. (2013). Origin of human colour preference for food. Journal of Food Engineering, 119, 508-515. DOI: 10.1016/j.jfoodeng.2013.06.021.

Lee, Y.; Lu, H. y Powers, J. (2005). Measurement of opalescence of resin composites. Dental Materials, 21, 1068-1074. DOI: 10.1016/j. dental.2005.03.015.

López-de-Alba, P. L.; López-Martínez, L.; Wrobel-Kaczmarczyk, K.; Wrobel-Zasada, K. y Amador-Hernández, J. (1997). Simultaneous determination of uranium (VI) and thorium (IV) ions with arsenazo III by partial least squares method. Journal of Radioanalytical and Nuclear Chemistry Letters, 220, 167171. DOI: 10.1007/BF02034851.

Michalski, R. (2014). The influence of color grouping on users' visual search behaviour and preferences. Displays, 35, 176-195. DOI: 10.1016/j.displa.2014.05.007.

Mollica, P. (2013). Color theory. Irvine CA, United States: Walter Foster.

Panush, S. (1993). Composiciones de pinturas opalescentes para automóviles que contienen pigmento de dióxido de titanio micronizado. No. de publicación: ES 2042 598. Oficina Española de Patentes y Marcas.

Park, J. G. (2013). Correlations Between Color Attributes and Children's Color Preferences.
Color Research and Application, 39(5), 452462. DOI: 10.1002/col.21801.

Prado-León, L. R. (2015). Color preferences in household appliances: data for emotional design. Procedia Manufacturing, 3, 5707-5714. DOI: 10.1016/j.promfg.2015.07.798.

Puebla, C. (2006). Whiteness assessment: a primer. Concepts, determination and control of perceived whiteness. Germany: Axiphos GmbH. Recuperado de http:/www.axiphos.com/ WhitenessPrimer.pdf

Schloss, K. B. (2015). Color preferences differ with variations in color perception. Trends in Cognitive Sciences, 19(10), 554-555. DOI: 10.1016/j.tics.2015.08.009.

Schloss, K. B. y Palmer, S. E. (2017). An ecological framework for temporal and individual differences in color preferences. Vision Research, in press. DOI: 10.1016/j.visres.2017.01.010.

Tutak, M.; Demiryürek, O. y Bulut, S. (2013). Effect of xenon arc irradiation on optical whitened polyester woven fabrics. Color Research and Application, 38(1), 73-78. DOI: 10.1002/ col.20727.

Ulusoy, B. y Olguntürk, N. (2017). Understanding responses to materials and colors in interiors. Color Research and Application, 42(2), 261272. DOI: $10.1002 / \mathrm{col} .22072$.

Vladusich, T. (2012). Simultaneous contrast and gamut relativity in achromatic color perception. Vision Research, 69, 49-63. DOI: 10.1016/j. visres.2012.07.022.

Yi, E. y Shamey, R. (2015). Crosscultural comparison of color terms and preference of persimmon-dyed fabric, galchon. Color Research and Application, 40(6), 592-604. DOI: 10.1002/col.21941.

Yu, L.; Westland, S.; Li, Z.; Pan, O.; Shin, M. J. y Won, S. (2017). The role of individual colour preferences in consumer purchase decisions. Color Research and Application, 42, 1-10. DOI: $10.1002 / \mathrm{col} .22180$.

\section{(ब) $\oplus \Theta \Theta$}

Análisis cromático y espectral de lacas acrílicas para retoque de pintura automotriz blanca mediante Reconocimiento de Pautas no Supervisado (Judith Amador- Hernandez y otros) por Revista Uniciencia se encuentra bajo una Licencia CreativeCommons Atribución-NoComercial-SinDerivadas 3.0 Unported. 